\title{
Der Mensch in der Personalarbeit
}

\section{A. Schreyögg}

Online publiziert: 20. Januar 2017

(C) Springer Fachmedien Wiesbaden 2017

Trotz der aktuell verstärkten Automatisierung gilt der Personalbereich in der Management-Literatur heute als zentrales Gebiet. In der Frühindustrialisierung, wo Organisationen als Maschinen-ähnliche Gebilde betrachtetet wurden, sah man die Werktätigen lediglich als Werkzeuge zur Zielerreichung. Das änderte sich erst in den 1950er- und 1960er-Jahren, als man die ökonomische Bedeutung eines qualifizierten Personals besser erkannte. Jetzt begann man so langsam, das Personal immerhin als wichtigen Produktionsfaktor zu begreifen. In vielen Unternehmen blieb aber der Einzelne nur ein Element des Personals, also des „Human Factors“. Bei dem Begriff „Personal“ handelt es sich ja um einen Agregatbegriff; das heißt, er meint die Gesamtheit aller Berufstätigen in einem organisatorischen System ohne Ansehen der einzelnen Persönlichkeiten. Dementsprechend befassten sich traditionelle Formen der Personalarbeit auch nicht mit den individuellen Belangen der berufstätigen Menschen. Es ging vielmehr um die „Gewinnung von Personal“, um dessen Führung, um die Entwicklung zur maximalen „Brauchbarkeit“ im System und um die Beurteilung dieses Personals, so wie es eben für die Funktionsfähigkeit einer jeweiligen Organisation relevant war.

In den 1970er-Jahren begannen zunehmend mehr Autoren, die Sicht vom Mitarbeiter als Werkzeug im Dienste der Organisation kritisch zu hinterfragen. In der Human Resources-Bewegung fragten sich manche Autoren sogar, ob Menschen in die üblichen Organisationsformen überhaupt integrierbar seien. In den meisten Systemen blieb aber alles wie bisher. Gegen Ende der 1980er-Jahre im Zuge neuer wirtschaftlicher Entwicklungen zeichnete sich dann aber doch zunehmend eine Tendenz ab, Berufstätige nicht mehr nur als Funktionsträger, sondern auch als Menschen mit ihren jeweiligen Stärken und Schwächen in den Blick zu nehmen. Daraus ergaben

Dr. A. Schreyögg $(\triangle)$

Breisgauer Str. 29, 14129 Berlin, Deutschland

E-Mail: info@schreyoegg.de 
sich dann auch ganz neuartige Formen der Personalentwicklung, der Führung und sogar der Personalauswahl. Und davon wird in dieser Ausgabe von OSC Manches berichtet.

Im ersten Beitrag stelle ich eine Palette von modernen Formaten rund um Training, Coaching usw. in den generellen Rahmen von Personalmanagement und Personalentwicklung. Stefanie Heinrich berührt mit ihrem Beitrag zum Life-Coaching nun ganz deutlich die individuelle Ebene, indem sie die Entwicklung von Lebenszufriedenheit durch Life-Coaching beforscht. Thomas Kretschmar und Moritz Senarclens de Grancy thematisieren personale Phänomene im Führungshandeln, und Sylke Langenbeck, Jens Nachtwei und Sebastian Uedelhoven beschäftigen sich mit Personalauswahl, die sie verantwortbar finden. Christine von Schachtmeyer zeigt in ihrem Praxisbericht, dass heute auch in Schulen individualisierende Beratung Einzug gehalten hat. Im Beitrag von Anke Larro-Jacob erhalten wir eine Kostprobe von der Bedeutung, die man heute der Gefährdungsbeurteilung psychischer Belastungen in Organisationen beimisst. Die nachfolgenden Beiträge thematisieren andere Phänomene: Haghiri Limoudehi zeigt uns, wie Märchen und Metaphern in der Supervision zu nutzen sind. Im ersten Diskurs beschäftigt sich Heike HanoldLynch, selbst Schauspielerin, mit Coaching von Schauspielern, bei dem Glaubwürdigkeit im Vordergrund steht. Der zweite Diskurs ist ein Beitrag von Beate WestLeuer über Literatur zum organisationsinternen Coaching.

\section{In eigener Sache}

Liebe Kolleginnen und Kollegen, liebe Leserinnen und Leser,

nachdem ich 1994 OSC ins Leben gerufen, dann 22 Jahre lang führend herausgegeben habe und jetzt selbst über das Pensionsalter hinaus gelangt bin, habe ich entschieden, die Herausgeberschaft in andere, in jüngere Hände zu übergeben. Ich freue mich besonders, dass Frau Prof. Heidi Möller bereit war, diese Aufgabe zu übernehmen. Denn sie ist nicht nur durch ihre bisherige fachliche Entwicklung prädisponiert, sondern auch durch ihre aktuelle Funktion als Lehrstuhlinhaberin für Beratungswissenschaften. Eine passendere Nachfolgerin hätte ich mir nicht vorstellen können, weshalb ich sie angesprochen habe, OSC unter ihre Fittiche zu nehmen. So weiß ich OSC in guten Händen. Denn das ist ja auch für mich keine Kleinigkeit, ein „wohl geratenes Kind“ wie die OSC, das über seine Teenagerzeit hinaus immer noch erfolgreich ist, ,adoptieren“ zu lassen. Jetzt werde ich mich aber aus der Führung von OSC gänzlich zurückziehen und nur noch als Mitglied des wissenschaftlichen Beirates fungieren.

Bei Bedarf stehe ich natürlich auch weiterhin mit Rat und Tat zur Verfügung. Für die Strategieentwicklung, die Zusammenstellung des Mitherausgeberstabes sowie des wissenschaftlichen Beirates, ist aber ab 2/2017 Frau Möller maßgeblich. Trotz meines Ausscheidens ist aber die Kontinuität von OSC gewahrt. Das liegt zum einen daran, dass Frau Möller schon seit etlichen Jahren als Mitherausgeberin Einfluss auf OSC nehmen konnte. Die Kontinuität ist aber besonders dadurch garantiert, dass Dr. Christoph Schmidt-Lellek, der seit den Anfängen von OSC Mitherausgeber ist 
und als Redakteur die Entwicklung dieser Zeitschrift maßgeblich mitgeprägt hat, diese Funktionen auch weiterhin wahrnehmen wird. Ich selbst werde nur noch als potentielle Autorin und als Beiratsmitglied OSC wohlwollend begleiten.

Jetzt bleibt mir nur noch, mich bei allen Kolleginnen und Kollegen, bei allen Leserinnen und Lesern, bei allen Autorinnen und Autoren sowie dem Verlagsleiter für Psychologie des Springer Verlags, Dr. Klockenbusch, für die ,gute Zeit mit OSC“" zu bedanken.

Dr. Astrid Schreyögg 